\title{
Magnetic confinement of the solar tachocline
}

\author{
Pascale Garaud \\ Departement of Applied Mathematics and Statistics, \\ Jack Baskin School of Engineering, \\ University of California at Santa Cruz, \\ California 95064, U.S.A
}

\begin{abstract}
Two distinct classes of magnetic confinement models exist for the solar tachocline. The "slow tachocline" models are associated with a large-scale primordial field embedded in the radiative zone. The "fast tachocline" models are associated with an overlying dynamo field. I describe the results obtained in each case, their pros and cons, and compare them with existing solar observations. I conclude by discussing new lines of investigation that should be pursued, as well as some means by which these models could be unified or reconciled.
\end{abstract}

\subsection{Introduction}

\subsubsection{Magnetic fields in the tachocline}

Two distinct possible origins for solar magnetic fields in the tachocline region can be identified. The ohmic decay timescale of a large-scale dipolar field embedded in the radiative interior is much larger than the estimated age of the Sun (Cowling 1945, Garaud 1999), so that a fraction of the magnetic flux initially frozen within the accreting protostellar gas is likely to persist today (Chapter 8). In parallel, according to the standard dynamo field theory, magnetic fields are thought to be constantly generated by fluid motions within the solar interior (Chapter 14). Optimal conditions for the generation of large-scale fields require the combination of large-scale azimuthal shear and small-scale helical motion, which are both naturally found in the region of the tachocline (Parker 1993; Ossendrivjer 2003; Tobias 2005; Chapter 14).

The fundamental differences between these two kinds of fields have naturally led to two distinct classes of tachocline confinement models: a slow tachocline, interacting on secular timescales with an underlying large-scale 
Table 9.1. Numerical values (in cgs units) of typical values of the density $\rho$, temperature $T$, gravity $g$, the buoyancy frequency $N$, the molecular magnetic diffusivity $\eta$, viscosity $\nu$, and thermal diffusivity $\kappa$ and finally the radius of the base od the convection zone $r_{\mathrm{cz}}$.

\begin{tabular}{cccc}
\hline Quantity & Value (in MKS) & Quantity & Value (in MKS) \\
\hline$\rho$ & 200 & $\eta$ & 0.043 \\
$\mathrm{~T}$ & $2.2 \times 10^{6}$ & $\nu$ & $2 \times 10^{-4}$ \\
$\mathrm{~g}$ & 530 & $\kappa$ & 1300 \\
$\mathrm{~N}$ & $9 \times 10^{-4}$ & $r_{\mathrm{cz}}$ & $5 \times 10^{8}$ \\
\hline
\end{tabular}

primordial field and slow meridional flows (Gough \& McIntyre 1998) and a fast tachocline, interacting on dynamical timescales with an overlapping or overlying dynamo field and small-scale turbulent flows (Forgács-Dajka \& Petrovay 2001). Historically the two types of models have remained clearly separated, so I shall take the same path and present them independently in Section 9.2 and 9.3. Whether the true tachocline genuinely does fall into one category or another has been widely debated during the meeting, and is discussed in Section 9.4 (see also Chapter 5).

\subsubsection{Characteristic numbers in the tachocline}

In order to compare models and observations of the tachocline, I adopt characteristic values for certain quantities in that region as listed in Table 9.1, which have been calculated by Gough (2006, this volume). For consistency, these values are used throughout this review; in some cases, however, they differ from those adopted by various other authors by factors up to a few.

\subsection{Primordial field confinement: the slow tachocline}

\subsubsection{First models}

With tremendous insight into today's debate, Mestel (1953) realised early on that even a very weak large-scale primordial field within the solar interior would have a significant impact on the solar angular velocity profile. Indeed, Alfvén waves are possibly the most efficient transporter of angular momentum in a rotating magnetized fluid. They propagate unimpeded along poloidal field lines with a characteristic velocity that depends on the local field amplitude and the local fluid density. Both the field geometry and the density stratification result in spatial inhomogeneities of the Alfvén ve- 
locity and the consequent phase mixing and damping of the waves. Angular momentum is then redistributed along (and across) the field lines, leading to a rotation profile satisfying Ferraro's isorotation law (Ferraro 1937)

$$
\mathbf{B} \cdot \nabla \Omega=0
$$

or in other words, with $\Omega$ constant along magnetic field lines. It has been argued that field amplitudes as low as $10^{-6} \mathrm{~T}$ are capable of enforcing uniform rotation to the entire radiative interior (Mestel 1953, Cowling 1957, Mestel \& Weiss, 1987).

The first model to study quantitatively the effect of an internal primordial field on the solar radiative zone rotation profile, and in particular its potential role in confining the tachocline, was proposed by Rüdiger \& Kitchatinov (1997). Shortly afterward, MacGregor \& Charbonneau (1999) complemented their work by studying the effects of different internal field geometries.

Both investigations evaluate the steady-state outcome of the interaction between a primordial field and the latitudinal shear diffusing from the convection zone. Meridional flows are assumed to be negligible, on the grounds that the strong local stratification effectively reduces their amplitude to a few centimeters per second (Miesch \& Gilman 2004); given this assumption, the poloidal component of the field decouples from the governing equations and can be chosen arbitrarily. While Rüdiger \& Kitchatinov only consider poloidal fields entirely confined within the radiative zone, MacGregor \& Charbonneau also study various cases in which at least some field lines overlap the convective zone. The steady-state structure of the toroidal field $B_{\phi}$ and angular velocity of the fluid $\Omega$ is then obtained by solving the azimuthal component of the momentum equation (here, cast in the form of a conservation equation for angular momentum) and of the induction equation.

$$
\begin{aligned}
& \nabla \cdot\left(\rho \nu r^{2} \sin ^{2} \theta \nabla \Omega\right)+\frac{1}{\mu_{0}} B_{\mathrm{p}} \cdot \nabla\left(r \sin \theta B_{\phi}\right)=0, \\
& r \sin \theta \mathbf{B}_{\mathrm{p}} \cdot \nabla \Omega+\eta\left(\nabla^{2} B_{\phi}-\frac{B_{\phi}}{r^{2} \sin ^{2} \theta}\right)=0,
\end{aligned}
$$

where the poloidal component of the field, $\mathbf{B}_{\mathrm{p}}$, is fixed. These equations are subject to the boundary conditions at the interface with the convective zone

$$
\begin{array}{r}
\Omega\left(r_{\mathrm{cz}}, \theta\right)=\Omega_{\mathrm{eq}}\left(1-a_{2} \cos ^{2} \theta-a_{4} \cos ^{4} \theta\right), \\
B_{\phi}\left(r_{\mathrm{cz}}, \theta\right)=0,
\end{array}
$$

where $\Omega_{\mathrm{eq}}, a_{2}$ and $a_{4}$ are derived from helioseismic inversions of the rotation 
profile in the convective zone; typically, $\Omega_{\mathrm{eq}} / 2 \pi=460 \mathrm{nHz}, a_{2}=0.14$ and $a_{4}=0.15$ (Charbonneau et al. 1999). Adequate regularity conditions are applied on the polar axis and at the centre. The boundary condition on the toroidal field is related to the assumption that any toroidal field at the interface with the convection zone is promptly removed through buoyancy instabilities. An alternative boundary condition that is sometimes also used assumes the convection zone to be an excellent insulator (with $\eta \rightarrow \infty$ ), and matches the interior field to a potential field. These two possibilities result in different quantitative estimates for the confining field strength and toroidal field amplitudes, but have otherwise qualitatively similar associated solutions.

The numerical solutions reveal a striking difference in angular velocity profile between the confined field and open field cases (see Figure 9.1). While the former results in a more-or-less uniformly rotating radiative zone, with a thin shear layer operating the smooth diffusive transition to the differentially rotating convective zone, the latter results in a latitudinally sheared state close to Ferraro isorotation, as field lines connected to the differentially rotating convection zone provide a support for the inward propagation of Alfvén waves. Helioseismic observations appear to set imperative constraints on the geometry of an embedded primordial field.

The angular momentum equation (9.2) illustrates the balance between viscous transport and magnetic transport near the outer boundary. A boundary analysis provides useful quantitative estimates of the tachocline properties in both open and confined geometries: viscous effects are only important in a thin Ekman-Hartmann boundary layer (see the review by Acheson \& Hide 1973) of width

$$
\delta_{\|}=\left(\frac{\mu_{0} \rho \nu \eta}{B_{0}^{2} r_{\mathrm{cz}}^{2}}\right)^{1 / 4} r_{\mathrm{cz}}=\left(\frac{E_{\nu} E_{\eta}}{\Lambda}\right)^{1 / 4} r_{\mathrm{cz}} \sim 4 \times 10^{-7} B_{0}^{-1 / 2} r_{\mathrm{cz}},
$$

where the field of amplitude $B_{0}$ is assumed to be mostly parallel to the outer boundary (as in the case of the confined field) and is measured in Tesla. The usual Ekman numbers are defined as

$$
E_{\nu}=\frac{\nu}{r_{\mathrm{cz}}^{2} \Omega_{0}}, E_{\eta}=\frac{\eta}{r_{\mathrm{cz}}^{2} \Omega_{0}},
$$

and a new parameter $\Lambda$ is defined as

$$
\Lambda=\frac{v_{\mathrm{A}}^{2}}{v_{\Omega}^{2}},
$$

where $v_{\Omega}=r_{\mathrm{cz}} \Omega_{0}$ and $v_{\mathrm{A}}$ is the Alfvén velocity $v_{\mathrm{A}}=B_{0} / \sqrt{\rho \mu_{0}}$. Here, $\Omega_{0}$ is a mean angular velocity of the system. This result is the first of 

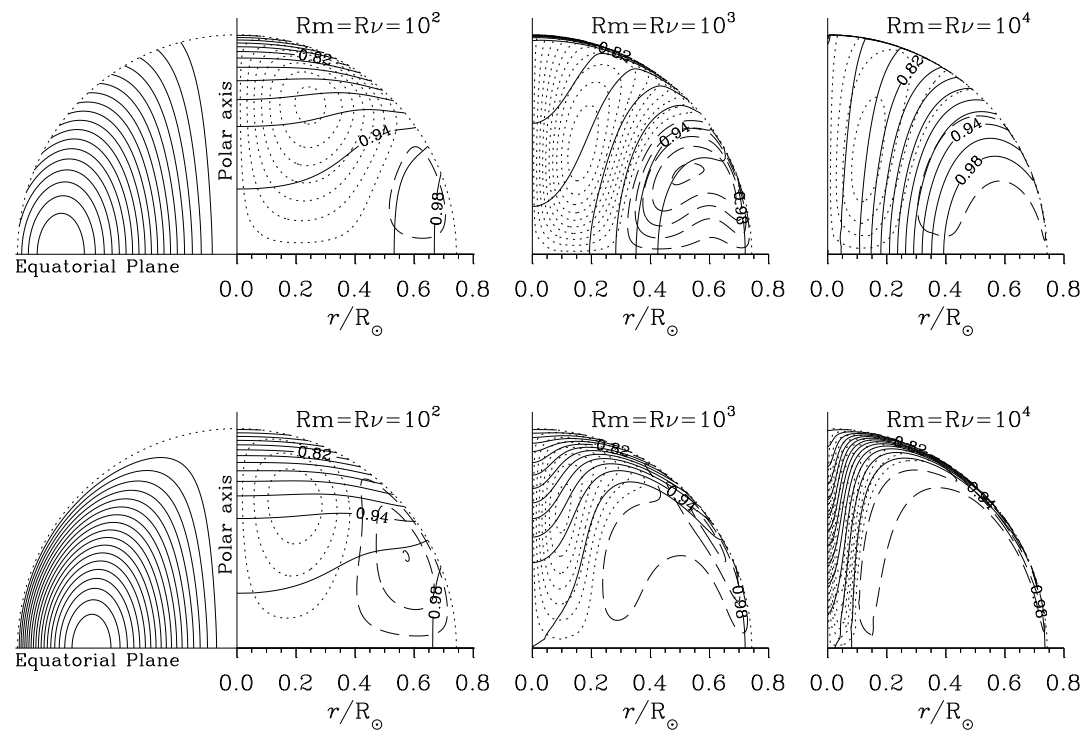

Fig. 9.1. Steady-state solutions obtained by MacGregor \& Charbonneau (1999) for the open and confined field configurations (top and bottom row respectively), for increasing Reynolds numbers. For this figure, the Reynolds numbers are defined as $R_{\nu}=B_{0} r_{\mathrm{cz}} /(\nu \sqrt{4 \pi \rho})$ and $R_{\mathrm{m}}=B_{0} r_{\mathrm{cz}} /(\eta \sqrt{4 \pi \rho})$, so that increasing the Reynolds numbers can be interpreted as increasing $B_{0}$ or decreasing $\nu$ and $\eta$. The left-side quadrant shows the poloidal field lines, whereas the right-side quadrants show the rotation profile (solid lines) and the toroidal field amplitude (dashed and dotted lines correspond to positive and negative $B_{\phi}$ ).

many estimates of the relation between the internal field strength and the tachocline thickness. If the poloidal field is given by

$$
\mathbf{B}_{\mathrm{p}}=\nabla \times\left(\frac{A}{r \sin \theta} \hat{\mathbf{e}}_{\phi}\right) \text { with } A=B_{\mathrm{in}} \frac{r^{2}}{2}\left(1-\frac{r}{r_{\mathrm{cz}}}\right)^{q},
$$

where the index $q$ controls the field concentration towards the interior, and $B_{\text {in }}$ is the amplitude of the field deep in the interior, then a field of amplitude $B_{0}$ in a tachocline of thickness $\Delta$ corresponds to

$$
B_{\text {in }} \simeq \frac{q}{2} B_{0}\left(\frac{r_{\mathrm{cz}}}{\Delta}\right)^{q-1}
$$

Combining all of the above estimates suggests that $2 \times 10^{-10} \mathrm{~T}$ near the edge of the convective zone (which corresponds to an interior field of about $6 \times 10^{-4} \mathrm{~T}$ for $q=5$ ) would confine the tachocline to its observed width of $0.03 r_{\mathrm{cz}}$ (Elliott \& Gough 1999). 
In a very interesting remark, MacGregor \& Charbonneau (1999) point out that even in a laminar tachocline, angular momentum transport between the convective and radiative zones would not, in fact, proceed through viscous effects only; as Spiegel \& Zahn (1992) had showed, the tachocline spread is aided by meridional flows, which approximately act as a hyperdiffusion of the kind

$$
\frac{\partial \Omega}{\partial t} \sim \frac{r_{\mathrm{cz}}^{4}}{t_{\mathrm{ES}}} \frac{\partial^{4} \Omega}{\partial r^{4}} \text { where } t_{\mathrm{ES}}=\frac{1}{4} \frac{N^{2}}{\Omega_{0}^{2}} \frac{r_{\mathrm{cz}}^{2}}{\kappa} \sim 2 \times 10^{11} \mathrm{yr},
$$

where $N$ is the local buoyancy frequency in the solar tachocline. In that case an equivalent boundary layer analysis reveals a different relation between the tachocline thickness and the field strength:

$$
\delta=\left(\frac{\mu_{0} \rho \eta}{B_{0}^{2} t_{\mathrm{ES}}}\right)^{1 / 6} r_{\mathrm{cz}}=\left(\frac{E_{\eta}}{\Lambda \Omega_{0} t_{\mathrm{ES}}}\right)^{1 / 6} r_{\mathrm{cz}} \sim 0.0001 B_{0}^{-1 / 3} r_{\mathrm{cz}} .
$$

The local poloidal field required to confine the tachocline is now of the order of $B_{0} \sim 6 \times 10^{-8} \mathrm{~T}$, and the resulting toroidal field has a typical amplitude of the order of $10 \mathrm{~T}$, which (as MacGregor \& Charbonneau point out) is interestingly close to the estimated upper limit for field storage against magnetic buoyancy within the tachocline (Schüssler et al. 1994).

\subsubsection{Towards a self-consistent model: the governing equations}

Despite the great degree of simplification inherent in the model just described, one essential result is of profound generality: Alfvénic angular momentum transport occurs on a very rapid timescale, and does not permit large deviations from isorotation anywhere in the radiative zone. Observed sheared regions (such as the tachocline) must be relatively free of poloidal field. The magnetic confinement problem takes an alternate but equivalent meaning: there must exist some mechanisms to confine the primordial field within the radiative zone in such a way as to be largely disjoint from the convective zone. Little overlap between the internal field and the convective region is also required by the upper limits set from observations of the sunspot parity throughout the cycles (Boyer \& Levy 1984, Boruta 1996).

The microscopic magnetic diffusivity in the radiative zone doesn't exceed $\sim 0.05 \mathrm{~m}^{2} \mathrm{~s}^{-1}$. Consequently, even very slow flows have a large magnetic Reynolds number. Radial motions in the tachocline are heavily suppressed by the strong local stratification, the flow speed for a steady-state system being controlled by the thermal diffusion time. Across the tachocline, the upper limit for the radial flow velocities is $u_{r} \sim 10^{-6} \mathrm{~m} \mathrm{~s}^{-1}$ with a corresponding magnetic Reynolds number of a few hundred, which is largely 
sufficient to have significant nonlinear interactions with the poloidal field contrary to the assumptions of the studies described in the previous section.

Gough \& McIntyre (1998) realized the importance of meridional flows in the dynamics of the tachocline. They proposed a model in which gyroscopic pumping near the convective-radiative interface drives flows whose role is to confine the interior field, thereby completing the missing piece of the tachocline puzzle.

Their model consists of four radially distinct regions (see Figure 9.2). In the convection zone (extended, if necessary, by a few tens of Mm to include the overshoot region, and a corresponding fast tachocline), angular momentum balance is achieved between anisotropic Reynolds or Maxwell stresses, and large-scale advection by meridional flows (zone 1). The flow geometry near the convective-radiative interface is dictated by the steady-state thermal wind and thermal energy balance. The flows burrow into the stably stratified, mostly laminar region directly underneath (zone 2) and interact with the deeply embedded magnetic field within a thin magnetic boundary layer (zone 3). This conveniently results in the simultaneous confinement of the underlying field to the lower part of the radiative zone, and that of the meridional flows within a well-ventilated but mostly magnetic free upper part of the radiative zone. Below the magnetic boundary layer, the confined field imposes uniform rotation to the bulk of the radiative zone (zone 4).

It is perhaps worth pointing out here that the notion of tachocline has largely evolved in recent years. Within the well-ventilated, magnetic-free region (zone 2) angular momentum is roughly conserved along the meridional flow lines and the latitudinal shear imposed by the convective zone is not so much suppressed as "reshuffled". As a result, given the strict definition of tachocline as "a strong shear layer beneath the convective region" one could argue that the Gough \& McIntyre tachocline is in fact limited to the magnetic diffusion layer. On the other hand, a more modern interpretation of the tachocline as "the region which operates the dynamical transition between the convection zone and the radiative zone" would then encompass both the magnetic diffusion layer and the magnetic-free region directly above. This distinction will be useful when comparing the various predictions for the tachocline thickness proposed in the literature. Moreover, a third meaning of tachocline confinement now emerges in relation to the tachocline meridional flows. Observations of surface abundances of light elements and helioseismic observation of the sound speed profile in the tachocline suggest that the depth of the mixed layer beneath the convection zone is of the order of a few percent of the solar radius (see Chapter 2 for more detail; Rüdiger \& Pipin, 2001; Elliott \& Gough 1999). Given that 


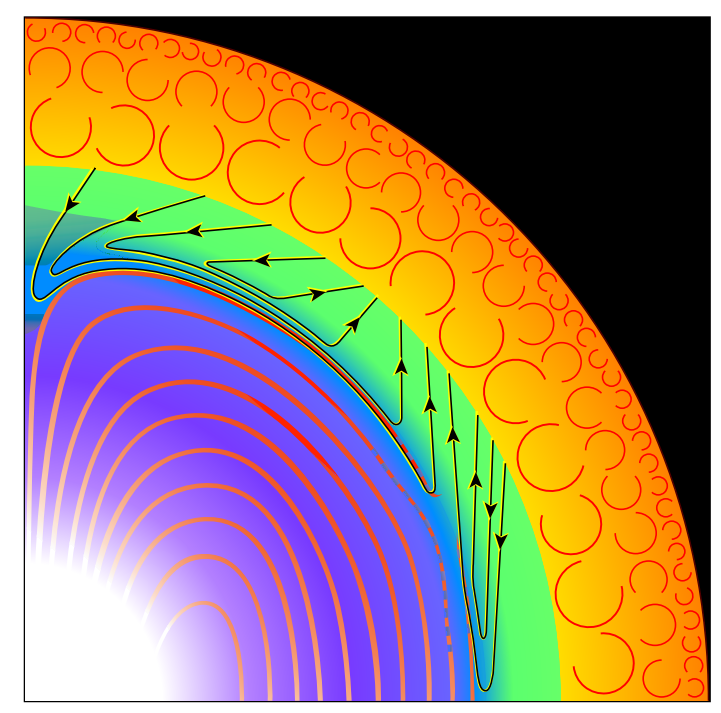

Fig. 9.2. Schematic representation of the Gough \& McIntyre model. The outer convection zone is differentially rotating, and generates meridional flows (black lines) through gyroscopic pumping. These confine the underlying field (thick grey lines) to the radiative interior, while leaving the tachocline virtually magnetic free.

the upper limits on the depth of the overshoot region have been recently estimated to be significantly smaller than the tachocline depth (Brummell, Clune \& Toomre 2002, Rogers \& Glatzmaier 2005) these observations can be related with reasonable confidence to the tachocline ventilation depth (zone 2 and 3 ).

The equations governing laminar fluid motions in the radiative zone consist of the momentum equation, the mass conservation equation, the thermal energy conservation equation, the field advection-diffusion equation, the equation of state and finally, a solenoidal condition for the field. When considering slow meridional flows in a slowly rotating star like the Sun, one can linearize the equations around a uniformly rotating, spherically symmetric background hydrostatic equilibrium and use the anelastic approximation. The complete set of equations representing the secular laminar dynamics of the radiative interior is then

$$
\begin{aligned}
& \rho \frac{\partial \mathbf{u}}{\partial t}+2 \rho \boldsymbol{\Omega}_{0} \times \mathbf{u}=-\nabla \tilde{p}-\tilde{\rho} \mathbf{g}+\mathbf{j} \times \mathbf{B}+\nabla \cdot \boldsymbol{\pi}, \\
& \nabla \cdot(\rho \mathbf{u})=0, \\
& \rho T \frac{\partial s}{\partial t}+\rho T \mathbf{u} \cdot \nabla s=\nabla \cdot(k \nabla T),
\end{aligned}
$$




$$
\begin{aligned}
& \frac{\tilde{p}}{p}=\frac{\tilde{\rho}}{\rho}+\frac{\tilde{T}}{T}, \\
& \frac{\partial \mathbf{B}}{\partial t}=\nabla \times(\mathbf{u} \times \mathbf{B})+\nabla \times(\eta \nabla \times \mathbf{B}), \\
& \nabla \cdot \mathbf{B}=0,
\end{aligned}
$$

where tildes denote perturbations from hydrostatic equilibrium, $s$ is the entropy, $\pi$ is the viscous stress tensor, $k=\rho c_{\mathrm{p}} \kappa$ is the thermal conductivity and all other quantities have their usual meaning. This complete system of equations has not been solved exactly yet for realistic solar values of the background state quantities. Numerical solutions have difficulties reaching simultaneously the correct thermal, viscous and magnetic diffusivities, while analytical solutions struggle to cope with the complex geometry and the intrinsic nonlinearity of the problem. What follows describes the various attempts at treating the problem that have been proposed so far.

\subsubsection{The Gough \& McIntyre boundary layer analysis}

The insight of Gough \& McIntyre's seminal work (1998) is to reduce the above system of equations to a boundary layer analysis, by considering from the outset the thin nature of the tachocline, and retaining in each zone identified the dominant terms in the dynamical balance only.

Thermal-wind balance in the upper region of the tachocline (zone 2). In this region, Gough \& McIntyre assume that the amplitude of the confined internal magnetic field is too low to have any significant effect on the flow dynamics. In that case, thermal-wind balance is achieved: the azimuthal component of the vorticity equation reduces to

$$
2 \Omega_{0} r \sin \theta \frac{\partial \tilde{\Omega}}{\partial z}=\frac{g}{r T} \frac{\partial \tilde{T}}{\partial \theta},
$$

where the pressure fluctuations in the equation of state have been neglected in accordance with the anelastic approximation. Maintaining the thermalwind balance against diffusion requires heat and momentum advection by meridional flows; within a thin-tachocline this is equivalent to:

$$
\frac{N^{2} T u_{r}}{g}=\frac{1}{\rho c_{\mathrm{p}} r^{2}} \frac{\partial}{\partial r}\left(r^{2} k \frac{\partial \tilde{T}}{\partial r}\right),
$$

where $k$ is the thermal conductivity $(\kappa=k / \rho c)$.

Additional information on the flow geometry related to the tachocline shear can be deduced qualitatively from equations (9.19) and (9.20). The 
observed angular velocity profile in the tachocline (as given by equation (9.4)) corresponds to a significant latitudinal entropy perturbation, positive near the poles and equator, and negative at mid-latitudes. In order to maintain this gradient against diffusion (specifically in the radial direction, since the overlying convective zone is largely isentropic), meridional flows are required with downwelling near the poles, and upwelling in mid-latitude. This geometry favours the internal field confinement only if the upwelling region is sufficiently narrow.

Advection-diffusion balance in the magnetic diffusion layer (the tachopause, zone 3 ). In the downwelling regions, the tachocline flow meets the underlying field and confines it to the radiative interior. In a steady state, the system is in equilibrium when the downward advection exactly compensates the outward diffusion of the field. Within a thin diffusion layer, the dominant terms of the advection-diffusion balance are extracted to yield

$$
\begin{aligned}
2 \Omega_{0} u_{\theta} \cos \theta= & \frac{B_{0}}{\mu_{0} \rho r \sin \theta} \frac{\partial}{\partial \theta}\left(B_{\phi} \sin \theta\right), \\
& -B_{0} \sin \theta \frac{\partial \tilde{\Omega}}{\partial \theta}=\eta \frac{\partial^{2} B_{\phi}}{\partial r^{2}},
\end{aligned}
$$

from the angular momentum and the azimuthal component of the induction equation respectively. Here, $B_{0}$ is the amplitude of the latitudinal component of the primordial field in the region of the tachocline. In addition, a rough estimate of the radial flow velocity required to balance the diffusion of the field in the boundary layer of thickness $\delta_{3}$ is

$$
u_{r} \sim \frac{\eta}{\delta_{3}}
$$

which can be combined with the anelastic mass conservation equation to obtain an estimate of the latitudinal velocity:

$$
\frac{1}{r^{2}} \frac{\partial}{\partial r}\left(r^{2} \rho u_{r}\right)+\frac{1}{r \sin \theta} \frac{\partial}{\partial \theta}\left(\rho \sin \theta u_{\theta}\right)=0
$$

Boundary layer scaling. Boundary layer scalings are easily derived using the approximations $\partial / \partial r \sim 1 / \delta_{2}$ in zone $2, \partial / \partial r \sim 1 / \delta_{3}$ in zone 3 and $\sin \theta \sim \cos \theta \sim 1 / \sqrt{2}$ with $\partial / \partial \theta \sim i L$, where $L$ is a latitudinal wavenumber.

Before outlining the results obtained by Gough \& McIntyre, it should be noted that in the limit where the magnetic diffusion layer is of similar width as the whole tachocline (in that case, there is no magnetic-free region - zone 2 and zone 3 are combined) $\delta_{2}=\delta_{3}$ and the combination of equations (9.19) 
to $(9.24)$ with $\partial / \partial r=1 / \delta$ yields (as expected) exactly the estimate of the tachocline thickness (9.12) derived by MacGregor \& Charbonneau (1999). For this scaling to hold, it is important to verify that the Lorentz force in the vorticity equation can be neglected compared to the thermal-wind balance. This is indeed the case for the field amplitude corresponding to the observed tachocline width.

The Gough \& McIntyre model suggests that a different force balance can occur when the magnetic diffusion layer is significantly thinner than the magnetic-free region. In zone 2 , a unique expression relating the flow amplitude and the thickness of the region $\delta_{2}$ can be derived from the thermalwind balance and the thermal energy equations, namely (9.19) and (9.20):

$$
u_{r} \sim \frac{2}{L}\left(\frac{\kappa}{r_{\mathrm{cz}}^{2} \Omega_{0}}\right)\left(\frac{r_{\mathrm{cz}}}{\delta_{2}}\right)^{3}\left(\frac{\Omega_{0}}{N}\right)^{2}\left(\frac{\tilde{\Omega}}{\Omega_{0}}\right) r \Omega_{0} .
$$

Note that if $\delta_{2}$ is fixed, this equation provides a stringent relation between the imposed shear and the meridional flows permitted within the tachocline.

Two scenarios may then occur depending on the strength of the internal field. The Gough \& McIntyre model assumes that the magnetic field amplitude within the tachopause is sufficiently small for the thermal wind relation to hold there as well. Thus, equations (9.19) and (9.20) complement equations (9.21), through to (9.24) in zone 3 to yield the scaling:

$$
\delta_{3} \sim\left(\frac{4}{L^{4}} \frac{v_{\Omega}^{2}}{v_{\mathrm{A}}^{2}} \frac{\Omega_{0}^{2}}{N^{2}} \frac{\kappa \eta}{r_{\mathrm{cz}}^{4} \Omega_{0}^{2}}\right)^{1 / 6} r_{\mathrm{cz}} .
$$

Note that the Gough \& McIntyre tachopause is exactly the boundary layer studied by MacGregor \& Charbonneau (1999) (see equation (9.12). Matching the tachopause dynamics with the overlying flow from zone 2 (by combining (9.26) with (9.23) and (9.25)) yields a unique relation between $\delta_{2}$ and $B_{0}$ :

$$
\delta_{2} \sim\left[\frac{2^{8}}{L^{10}} \frac{v_{\Omega}^{2}}{v_{\mathrm{A}}^{2}}\left(\frac{\kappa}{\eta}\right)^{5} \frac{\kappa^{2}}{r_{\mathrm{cz}}^{4} \Omega_{0}^{2}}\left(\frac{\Omega_{0}^{2}}{N^{2}}\right)^{7}\left(\frac{\tilde{\Omega}}{\Omega_{0}}\right)^{6}\right]^{1 / 18} r_{\mathrm{cz}} .
$$

Comparing the expression for $\delta_{2}$ to the observed tachocline ventilation depth as measured by Elliott \& Gough (1999), Gough \& McIntyre deduce that the internal field strength (in the tachocline region) is of the order of $10^{-4} \mathrm{~T}$, corresponding to a primordial field strength in the deep interior of the Sun of the order of $1 \mathrm{~T}$. As assumed, the thickness of the tachopause is only a few percent of the thickness of the whole tachocline. The tachocline ventilation time is of the order of $3 \times 10^{6} \mathrm{yrs}$; while being slow, it provides sufficient 
mixing of light elements beneath the convective zone to explain the observed abundances of $\mathrm{Li}$ and $\mathrm{Be}$. This ventilation timescale is yet significantly smaller than the solar spin-down timescale, which accounts for the fact that the interior angular velocity is close to that of the surface layers.

Given this estimate for the field amplitude in the tachopause, it appears that neglecting the Lorentz force in the vorticity equation is only marginally justified. In fact, Gough \& McIntyre themselves acknowledge that the thermal-wind relation may not hold in the lower regions of the magnetic boundary layer, where the nonlinear interaction between the field and the flow is maximal. What happens in the alternative case has not been evaluated in detail yet; however, dropping equations (9.19) and (9.20) plausibly describes the right balance, and reveals a new boundary layer scaling

$$
\delta_{3} \sim\left(\frac{2}{L^{3}} \frac{\Omega_{0}}{\tilde{\Omega}} \frac{v_{\Omega}^{2}}{v_{\mathrm{A}}^{2}} \frac{\eta^{2}}{r_{\mathrm{cz}}^{4} \Omega_{0}^{2}}\right)^{1 / 4} r_{\mathrm{cz}},
$$

which, when combined with equation (9.23) from the poloidal advectiondiffusion balance, and equation (9.25) from thermal-wind balance in zone 2 reveals yet another possible relation between the tachocline thickness, the imposed shear and the magnetic field:

$$
\delta_{2} \sim\left[\frac{2^{5}}{L^{7}}\left(\frac{\kappa}{\eta}\right)^{4} \frac{\eta^{2}}{r_{\mathrm{cz}}^{4} \Omega_{0}^{2}}\left(\frac{\Omega_{0}^{2}}{N^{2}}\right)^{4}\left(\frac{\tilde{\Omega}}{\Omega_{0}}\right)^{3} \frac{v_{\Omega}^{2}}{v_{\mathrm{A}}^{2}}\right]^{1 / 12} r_{\mathrm{cz}} .
$$

The main difference between this boundary layer analysis and the one proposed by Gough \& McIntyre is the non-thermal nature of the boundary layer. $\dagger$

So which (if any) of the above scalings really correspond to the solar tachocline? This question is difficult to answer without a careful quantitative estimate of the force balance in the tachopause, which can only be done through numerical simulations. Moreover, since the Coriolis force and the field geometry vary strongly with latitude, the force balance and the nature of the boundary layer is very likely to differ between the equator, midlatitudes and the poles.

\subsubsection{Numerical solutions of the Gough \& McIntyre model}

To obtain a more precise view of the geometry of the tachocline dynamics, as well as quantitative predictions for the internal rotation rate, the light

$\dagger$ The tachopause in the Gough \& McIntyre model is also a thermal boundary layer. 
element depletion timescale and the amount of overlap between the interior field and the convective zone, one must resort to numerical simulations. Two approaches have recently been considered. Douglas Gough and I (Garaud 2002; Garaud \& Gough 2006) have been interested in studying the steady-state tachocline balance, while Brun \& Zahn (2006) are looking at its temporal evolution for a given initial poloidal field configuration. While the former is able to bypass the various numerical problems caused by the wide range of timescales inherent in the physics of the system, the latter is ideally suited to the study of potential multiple equilibria, and naturally eliminates from the force balance any processes occurring on a timescale longer than the stellar evolution timescale.

\subsubsection{Steady-state calculations}

Axially symmetric steady-state calculations can be performed by an expansion of all governing equations on a suitably selected basis of orthogonal polynomials in the latitudinal direction, and then by solving the remaining ODEs using a Newton-Raphson relaxation procedure. Note that other methods also exist (expansion in spherical harmonics or finite differences in all directions), but have not been implemented for the steady-state problem.

In 2002, I presented a preliminary numerical study of the nonlinear interaction between the primordial field and the meridional flows, in an idealized setup where the solar tachocline and radiative zone are assumed to be composed of an incompressible, homogeneous and isentropic fluid (Garaud 2002). This assumption largely simplifies the set of governing equations since all thermodynamical quantities decouple from the system; however, it also eliminates the crucial baroclinicity that is thought to drive meridional flows. These must then be artificially replaced by Ekman flows driven by viscous forces on a no-slip impermeable boundary. The latitudinal variation of the Coriolis force implied by the imposed shear from the convection zone (for the Gough \& McIntyre model) and in a viscous Ekman layer (in the simplified model) provides gyroscopic pumping with a similar geometry, but of different amplitude. This simplified model could clearly not provide any quantitative estimates of the tachocline dynamics but the geometrical similarities with the correct model provide an interesting complement to the Gough \& McIntyre (1998) boundary layer analysis.

In this simplified model, the equations solved are the following:

$$
\begin{aligned}
& 2 \boldsymbol{\Omega}_{0} \times \mathbf{u}=-\nabla p+\mathbf{j} \times \mathbf{B}+\nu \nabla^{2} \mathbf{u}, \\
& \nabla \cdot \mathbf{u}=0, \\
& \nabla \times(\mathbf{u} \times \mathbf{B})=\eta \nabla \times(\nabla \times \mathbf{B}),
\end{aligned}
$$




$$
\nabla \cdot \mathbf{B}=0
$$

with a fiducial density $\rho=1$. No-slip, impermeable boundary conditions are assumed for the meridional flows, and on the upper boundary the rotation profile is given by the convection zone profile (see equation 9.4). The lower boundary is a stress-free solid conducting core. The field is matched onto a potential field decaying exponentially outside the radiative zone, and matching on to a point dipole of given amplitude $B_{\text {in }}$ located at the center of the inner core.

The dynamical connection between the interior flow and the top boundary operates through Ekman and Hartmann layers, which have typical scalings of the order of

$$
\delta_{\nu}=E_{\nu}^{1 / 2} r_{\mathrm{cz}},
$$

for a purely viscous Ekman layer, and

$$
\delta_{\|}=\left(\frac{E_{\nu} E_{\eta}}{\Lambda}\right)^{1 / 4} r_{\mathrm{cz}} \text { and } \delta_{\perp}=\left(\frac{E_{\nu} E_{\eta}}{\Lambda}\right)^{1 / 2} r_{\mathrm{cz}}
$$

for Hartmann layers when a magnetic field of amplitude $B_{0}$ is respectively parallel and perpendicular to the outer boundary. Ekman numbers of the order of $10^{-5}$ or less are therefore required to model structures on the scale of the tachocline.

In what follows, it is important to remember that the induction equation is linear in the field amplitude; thus, the ability of the flow to confine the field depends not so much on the field amplitude as on the meridional flow velocity and corresponding magnetic Reynolds number $R_{m}=u_{r} \delta / \eta \dagger$. Gyroscopic pumping (of the Ekman, or Ekman-Hartmann type) on the outer boundary implies that the latitudinal component of the flow $u_{\theta}$ has amplitude comparable to the azimuthal velocity of the outer boundary $u_{\phi}$, whereas the radial component of the flow is given by $u_{r} \sim \delta u_{\theta} / r_{\mathrm{cz}}$ where $\delta$ is the thickness of the relevant boundary layer. This simple estimate has two important consequences. Since $\delta$ is naturally smaller for larger field strength, the stronger the field, the smaller the effective magnetic Reynolds number. Moreover, for a given field strength $\delta_{\|} \gg \delta_{\perp}$, so that the Ekman-Hartmann flow is much stronger in the confined field case (i.e. parallel to the outer boundary) than for the open field case (i.e. perpendicular to the outer boundary). The system is therefore subject to a strong positive feedback effect: when and where the field lines are confined because of an initially large flow amplitude, the resulting field geometry permits large flow amplitudes. The converse is true

$\dagger$ Note that there is, in this simulation, an indirect dependence on the field strength through Ekman-Hartmann pumping. 
for the open field case, with weak flows as a cause and consequence of the radial field geometry on the boundary. Such dual dynamics with positive feedback in both limits is likely to harbour multiple equilibria. Unfortunately, the numerical algorithm I use is not ideally suited for the search for co-existing steady states; this could however be the subject of an interesting investigation.

The following results are the only steady-states found for a given set of parameters. Varying the internal field strength (through $\Lambda$ ) for fixed Ekman numbers reveals three possible dynamical structures. Note that the physical interpretation of the numerical results given here differs from that of the original paper (Garaud 2002), and should be preferred.

For low field strengths $(\Lambda \ll 1)$, the internal flow is dominated by Coriolis forces, with a more-or-less cylindrical angular velocity profile (commonly referred to as Taylor-Proudman rotation). Meridional flows are of Ekman type (with $u_{r} \sim E_{\nu}^{1 / 2} r_{\mathrm{cz}} \tilde{\Omega}$ ), penetrate deep into the radiative zone, and confine the field to the interior (except in the polar regions).

For very high field strengths $(\Lambda \gg 1)$, the internal flow is dominated by Lorentz forces, and the angular velocity is in a state of isorotation with the field. In contrast with the previous case, the driven flows are particularly weak $\left(u_{r} \sim \delta_{\perp} \tilde{\Omega}\right.$, so that $\left.R_{m} \sim E_{\nu} / \Lambda \ll 1\right)$, and do not have significant effects on the field which retains a mostly dipolar structure throughout the computational domain. The field lines freely connect with the convective zone, and the shear is propagated inwards accordingly. In this limit, it is in fact possible to linearize the equations around a state of isorotation, which was successfully done by Dormy, Cardin \& Jault (1998) and Dormy, Jault \& Soward (2002).

For intermediate field strength, the nonlinear interaction between the internal field and the meridional flows dominates the dynamics of the interior. Two separate regions can be identified. The essentially radial geometry of the flow in the polar regions, as suspected by Gough \& McIntyre, provides only weak coupling with the underlying (mostly radial) field. Polar field lines are connected to the convection zone, which results in slowly rotating, strongly sheared polar regions. On the other hand, the downwelling flow near the equator is able to confine the internal field over a broad range of latitudes, which results in a uniform rotation profile below. In this region, a Hartmann layer is observed with flow amplitudes scaling as $u_{r} \sim \delta_{\|} \tilde{\Omega}$ and corresponding to a magnetic Reynolds number $R_{m} \sim\left(E_{\nu} / E_{\eta}\right)^{1 / 2} \Lambda^{-1}=P_{m}^{1 / 2} / \Lambda$ (where $P_{m}=\nu / \eta$ is the magnetic Prandtl number). The meridional flows themselves are deflected by the underlying field and the resulting radial mixing is 
strongly suppressed. There is a marginal hint for the type of nested boundary layer structure predicted by Gough \& McIntyre (1998), with a largely magnetic-free region overlying a thin diffusion layer. However, this result needs to be confirmed with lower diffusivity simulations.

The intermediate field strength case appears to approach qualitatively the dynamical structure that we may expect to see in the solar tachocline. However, the incompressible and isentropic nature of the fluid is an intrinsic flaw of this preliminary work which needs to be addressed. New results obtained by Douglas Gough and I on the steady-state structure of the Gough \& McIntyre tachocline including stratification and thermal diffusion were presented at the Isaac Newton meeting. This time, the complete set of equations (9.13) to (9.18) are solved for a steady-state solution. The boundary conditions are similar to the ones used in the incompressible case for the magnetic field, but the assumption of "impermeability" of the base of the convection zone to flows was dropped in favour of one which assumes the continuity of Reynolds stresses across the boundary. Several Reynolds stress prescriptions in the convection zone are currently being explored, and the preliminary results presented in Figure 9.3 correspond to a simplistic stress-free assumption. The main consequence of this new set of boundary conditions is to eliminate spurious Ekman flows and let the force balance within the tachocline dictate the flow amplitude and geometry.

The background state used was derived from a realistic solar model (Christensen-Dalsgaard, Gough \& Thompson 1991) where, for numerical purposes, the thermal conductivity, viscosity and magnetic diffusivity are artificially increased by the factors $f_{k}, f_{\nu}$ and $f_{\eta}$ respectively; this is necessary, since viscous and magnetic diffusion layers on the artificial outer boundary are otherwise too thin to be resolved. Typical values of $f$ achieved in preliminary simulations are of the order of $10^{7}$, with corresponding Ekman numbers of the order of $10^{-6}$; when $f_{\nu}=f_{\eta}=f_{\kappa}$ the solar values of the magnetic and thermal Prandtl numbers are respected.

In the absence of strong magnetic fields the amplitude and geometry of the meridional flow satisfy the expectations from the Gough \& McIntyre model: the steady-state solutions appear to depend on the thermal conductivity only, confirming that the weak flows that may be driven by the artifial stresses on the outer boundary are negligible compared to the baroclinic flows. These numerical results do therefore provide a good insight into the slow tachocline dynamics.

A thorough quantitative study of the numerical solutions is currently being performed, but preliminary qualitative results are found to be very sensitive to the thermal and magnetic diffusion parameters $f_{k}$ and $f_{\eta}$. According 
to the scalings obtained in Section 9.2.3, the magnetic Reynolds number corresponding to the tachocline ventilation flow is

$$
R_{m} \sim \frac{\kappa}{\eta} \frac{\Omega_{0}^{2}}{N^{2}} \frac{r_{\mathrm{cz}}^{2}}{\Delta^{2}} \frac{\tilde{\Omega}}{\Omega_{0}} \sim 0.01 \frac{r_{\mathrm{cz}}^{2}}{\Delta^{2}}
$$

for solar values of the diffusion and rotation parameters. Hence provided there exists a confining mechanism for the tachocline and $\Delta \ll r$ then $R_{m} \gg$ 1 , confirming the nonlinear interaction between the field and the flow; on the other hand, $R_{m} \ll 1$ if the tachocline is not confined. Again, this dual structure suggests either a very strong sensitivity of the equilibrium solution to the input parameters, or even the existence of multiple equilibria.

For most parameter values (in the low-diffusivity limit) numerical simulations show that the internal field retains a mainly dipolar structure with field lines connecting to the convective region. The interior rotation profile is close to a state of isorotation, and no tachocline is observed in this limit.

For carefully chosen parameters, however, it is possible to obtain solutions that are encouragingly close to what may be expected from a slow tachocline (see Figure 9.3). Meridional flows burrow into the radiative zone and confine the field to the interior except within the upwelling region. The width of the upwelling region is always of the order of the depth of the tachocline, and the flow direction within the upwelling region is roughly parallel to the rotation axis. Contrary to the incompressible simulations, field confinement also occurs in the polar regions. Interestingly, a thermal boundary layer appears to be present in the polar regions, but not in the equatorial regions.

\subsubsection{Time-dependent calculations}

The first numerical time-dependent simulations of a slow solar tachocline following the idea of Gough \& McIntyre were presented by Sacha Brun \& Jean-Paul Zahn at this meeting. The numerical algorithm used is the ASH code (Glatzmaier 1984; Clune et al. 1999, Miesch et al. 2000; Brun et al. 2004), which performs a spectral decomposition of the governing MHD anelastic equations on spherical harmonics and Chebishev polynomials in the horizontal and vertical directions respectively. The massively parallel numerical algorithm achieves significant resolution in all three directions. It is ideally suited for studying the radiative-convective interface.

Brun \& Zahn (2006) study numerically the dynamical evolution of the radiative zone when subject to shearing from the overlying convective region, and in the presence of a large-scale embedded primordial field. Their computational domain includes the radiative zone only, and they model the radiative-convective interface as an impermeable, electrically and ther- 

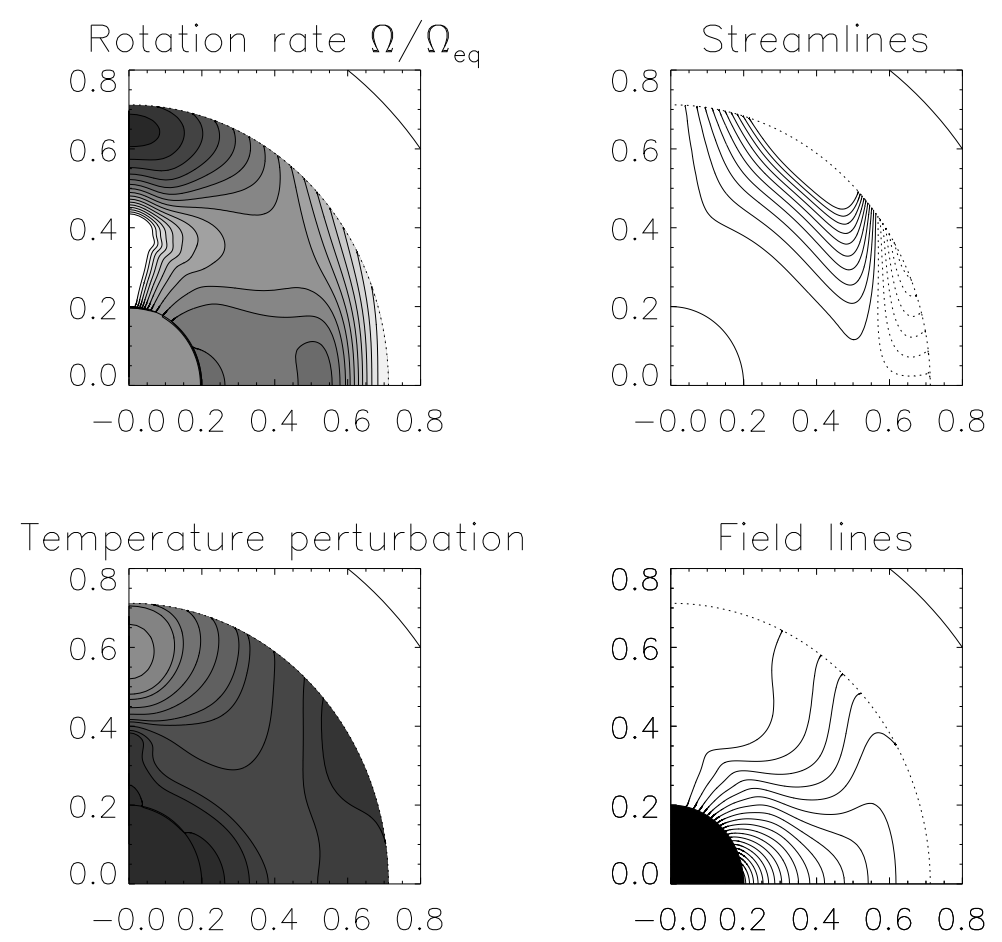

Fig. 9.3. Numerical results of equations (9.13) to (9.18) in a steady-state calculation for $f_{\nu}=f_{\kappa}=5 \times 10^{8}$ and $f_{\eta}=5 \times 10^{6}$. Each quadrant shows the solution in the radiative zone only, and the dotted line represents the edge of the convection zone. The rotation rate contours (from darker to lighter colors) range from $0.6 \Omega_{\mathrm{eq}}$ to $\Omega_{\mathrm{eq}}$. The streamlines are shown with dotted lines for clockwise flows and solid lines for anti-clockwise flows. The temperature perturbations range from $0 \mathrm{~K}$ to $+50 \mathrm{~K}$.

mally conducting, sheared boundary. Various initial magnetic field configurations are studied, ranging from deeply embedded fields to open field configurations. As an added bonus, the assumption of axial symmetry is dropped which enables them to study the emergence of all the possible non-axisymmetric MHD and baroclinic instabilities that have recently been discussed (Chapter 10 and 11, this volume), as well as the angular momentum transport from the associated Reynolds and turbulent Maxwell stresses (see Section 9.2.5.1).

The numerical values of the viscous, thermal and magnetic transport coefficients $(\nu, \eta$ and $\kappa)$ used in the ASH code are far greater than the microscopic solar values; however, by respecting their hierarchy (i.e. by respect- 
ing the hierarchy of all expected boundary layer widths and all dynamical timescales), Brun \& Zahn attempt to capture the essential dynamical balance in the tachocline, if not quantitatively at least qualitatively.

Their main result could reshape our view of the slow tachocline: none of the simulations appear to reach the steady-state balance suggested by the Gough \& McIntyre model. Instead, the system is observed to evolve in time following the diffusion of the magnetic field out of the radiative zone. In consequence, the dynamical evolution of the interior depends crucially on the initial magnetic configuration.

For initially open field lines, isorotation is rapidly achieved, as suspected from the results of MacGregor \& Charbonneau (1999). The meridional flows are strongly suppressed by the Lorentz force exerted by the mostly radial field lines, and fail to confine the field (the magnetic Reynolds number associated with their flows is of order of unity). After a rapid transient period (roughly, one Alfvén time), the system continues evolving as a result of the slow global field dissipation, whilst remaining in a Ferraro state. There is no evidence for the presence of a tachocline in this case.

When the field is initially in a configuration close to what one may expect from the Gough \& McIntyre steady state (corresponding to the marginally confined field configuration of Rüdiger \& Kitchatinov, 1997), one could expect that the meridional flows, not being hindered by the field, would act in such a way as to confine it (see the incompressible analogue discussed in the Section 9.2.4.1). However, Brun \& Zahn find that in this case also, the field lines quickly diffuse across the initially existing tachocline, connect to the convection zone and from there ensues Ferraro isorotation within a short Alfvénic timescale. It appears that although meridional flows of the kind predicted by Gough \& McIntyre are indeed observed in the simulation, they do not have enough time to achieve dynamical balance in the magnetic diffusion layer before the field diffuses and connects with the convective zone.

Only for a deeply confined initial field does the outward diffusion occur slowly enough to allow for the formation of the tachopause. In that case, magnetic field lines are indeed seen to be confined to the radiative interior by the meridional flows, except in the polar regions which retain a modest amount of latitudinal shear. This simulation appears to reproduce the Gough \& McIntyre view of the slow tachocline, save for a very important difference: the Ohmic diffusion of the internal field is only partially reduced by the tachocline dynamics, so that the field amplitude steadily decreases on a magnetic diffusion timescale. As this happens, the position and width of the tachocline and tachopause slowly change (the tachopause rises, and the tachocline becomes correspondingly thinner). 
The absence of a stable steady-state implies a direct relationship between the observed tachocline structure and the initial field configuration. This result, should it be confirmed, has important implications for dynamo action during the pre-main-sequence phase of solar evolution. A primordial centrally condensed magnetic field configuration can presumably only be achieved by a timely switch from a steady-state or largely irregular dynamo to a cyclic dynamo, which must happen before the convection zone has entirely retreated to its present radius. This idea is plausible given that the timescale for the evolution of the convective-radiative interface $\left(\sim 10^{7} \mathrm{yr}\right)$ is much shorter than the magnetic diffusion timescale $\left(\sim 10^{10} \mathrm{yr}\right)$. In addition, the Mount Wilson Ca II program has found strong observational evidence for a transition from irregular dynamo action in very young stars to periodic dynamos for older stars (Saar et al. 1994). This trend has been associated with the transition between very rapid young rotators and slower older rotators, and interestingly, the timescale for magnetic braking of very young stars is also of the order of $10^{7}$ yrs. Schüssler (1975), Parker (1981) and Mestel \& Weiss (1987) studied the typical magnetic fields that are likely to remain from dynamo action during the pre-main-sequence stage; perhaps it is time to revisit their results in the light of Brun \& Zahn's simulations using modern dynamo models, numerical algorithms and recent observations. Note that Chapter 8 reviews other recent ideas concerning the origin and stability of magnetic fields in the Sun.

However, the numerical results obtained by Brun \& Zahn pose another important problem. In all simulations, even for the most centrally condensed initial field configurations, the tachopause eventually reaches the outer boundary and, as field lines connect with the convective region, the system switches to the usual Ferraro state of isorotation. Using a rough scaling argument to compensate for the large diffusivities used in the simulations, Brun \& Zahn estimate that this state is likely to be achieved before the present solar age regardless of the initial conditions. This striking result is difficult to reconcile with helioseismic observations; if confirmed, it could shed serious doubts on the relevance of the current slow tachocline model to the solar radiative zone. However, I discuss in Section 9.2.5.3 how a better understanding of the outer boundary conditions to be applied to the slow tachocline model may rescue the situation.

\subsubsection{Discussion and prospects for slow tachocline models.}

In recent years, slow tachocline models have come under increased scrutiny and criticisms. By nature, they ignore phenomena occurring on rapid time- 
scales, concentrating instead on the secular dynamical interaction between slow meridional flows and the internal field. As such, they neglect three important effects that are likely to have significant impact on the fragile balance described above: the potential axisymmetric and non-axisymmetric instabilities of the calculated equilibria, the combined effects of all possible rapid-timescale angular-momentum transporters known to exist in the tachocline, and the effect of an overlying dynamo field. In addition, the typical boundary conditions used to model the interface with the convective zone are highly idealized and may distort our view of the tachocline. I shall now discuss briefly the consequences of these effects on our understanding of the tachocline dynamics.

\subsubsection{Stability of the slow tachocline models}

Slow tachocline models may be subject to a wide variety of instabilities, including purely hydrodynamical shear and baroclinic instabilities, MHD instabilities of the large-scale primordial field, magnetic buoyancy instabilities, magneto-rotational instabilities and magneto-shear instabilities. Detailed investigations in the context of the slow tachocline model are only just beginning.

Linear and quasi-linear stability analyses of an idealized purely hydrodynamical tachocline shear flow in the non-diffusive limit have been performed by Watson (1981), Charbonneau, Dikpati \& Gilman (1999), Dikpati \& Gilman (2001) and myself (Garaud 2001). The tachocline latitudinal shear is found to be close to marginal stability. The observed radial shear is stabilized by the very strong stratification (the typical Richardson number is of the order of a thousand). However, as Schatzman, Zahn \& Morel (2000) point out, the standard Richardson criterion for stratified shear instability must be corrected for thermal diffusion in the tachocline; in that case, the radial shear is again close to marginal stability. In addition, Petrovay (2003) suggests that independent shellular fluid motions create much stronger small-scale radial shear layers, which could lead to secondary shear instabilities in the tachocline. This interesting proposal has not been confirmed numerically yet, but would correspond to a scenario close to the one proposed by Spiegel \& Zahn (1992), and have important consequences for all slow and fast tachocline models alike.

In any case, the addition of magnetic fields changes the nature and stability of non-axisymmetric perturbations (Gilman \& Fox 1997, 1999a, 1999b; Dikpati \& Gilman 1999; Gilman \& Dikpati 2000; Cally, Dikpati \& Gilman 2003 and other publications by the same authors; Tobias \& Hughes 2004). A review of the stability of tachocline flows in the presence of strong fields is 
given in Chapters 10 and 11. However, the focus of most studies has largely been the stability of a dynamo-related magnetic field to shear and buoyancy instabilities. As such, the fields studied have idealised geometries (mostly toroidal) and high amplitudes ( $>1 \mathrm{~T}$ ) that do not resemble those typically encountered in slow tachocline models; their results are not directly applicable here. The magneto-rotational instability could operate in regions of the Sun where angular velocity decreases outward from the rotation axis (as it does in the polar regions). Balbus \& Hawley (1994) showed that the strong local stratification of the tachocline limits displacements to horizontal surfaces, as expected; this could provide a source of unaccounted for latitudinal momentum mixing in the polar regions.

Even more problematic for slow tachocline models are the well-known non-axisymmetric field instabilities of a mostly dipolar field in stellar interiors. Early works by Wright (1973), Markey \& Tayler $(1973,1974)$ and Pitts \& Tayler (1985) already suggested that a purely dipolar structure deep in the interior (as assumed in the above slow tachocline models) was subject to adiabatic perturbations near its neutral points (any confined field structure necessarily has such points). These are known to be stabilized by the presence of toroidal fields, but the current slow tachocline field structures are indeed found to be unstable (Brun \& Zahn 2006). A new method for finding possible stable field structures in stellar interiors was developed by Braithwaite \& Spruit (2004) and is outlined in Chapter 8. It would be interesting to see how the slow tachocline models may be modified by the additional constraint that the underlying primordial field should be in a stable configuration.

Self-consistent studies of the model and of its stability have tentatively been performed. The Newton-Raphson relaxation algorithm I have used to calculate steady-state solutions of the slow tachocline equations cannot find unstable equilibria. Therefore the solutions found for the range of diffusion parameters studied are known to be stable to all axisymmetric perturbations. However, it provides no information on the evolution of nonaxisymmetric perturbations. The numerical algorithm used by Brun \& Zahn (2006), on the other hand, is ideally suited for the study of 3D instabilities of all kind. They observe the growth of non-axisymmetric instabilities associated with the primordial dipolar field, but do not detect any other intrinsic instabilities in the tachocline region. This result is interesting in the light of the local and global analyses mentioned above, but could be consistent with instabilities that only develop at high Reynolds and magnetic Reynolds numbers.

In conclusion, there are clear signs that the slow tachocline model might 
be unstable to a variety of non-axisymmetric instabilities. These could play an important role in redistributing chemical species, angular momentum and thermal energy within the tachocline, and must therefore be analyzed. Various clues to the relative lack of mixing below the tachocline also suggest that any derived model should be constructed in such a way as to maximize stability below the tachocline; this constrains the geometry of the assumed primordial field.

\subsubsection{Gravity waves as angular momentum transporters}

The tachocline is known to host a wide spectrum of gravity waves, excited by overshooting convective plumes pounding on the stably stratified interior. These waves transport and deposit angular momentum further down in the radiative zone; the differential damping between prograde and retrograde waves is known to accentuate shearing flows and can be likened to some kind of anti-diffusion mechanism (McIntyre 2003, and Chapters 4 and 5; Kumar, Talon \& Zahn 1999; Kim \& MacGregor 2001, 2003; Talon \& Charbonnel 2005). Quantitative estimates for the amplitude of the gravity waves thus generated, as well as their damping rate as a result of nonlinear interactions (mode-mode interaction or critical layer interaction) are difficult to obtain, although numerical simulations provide a new promising route for resolving this problem (Rogers \& Glatzmaier 2006). To complicate matters, dynamical interactions between the gravity waves and magnetic fields in the tachocline transfer energy into a wider spectrum of Alfvén waves, with correspondingly different propagation and damping mechanisms (Kim \& MacGregor 2003). The global action of gravity and Alfvén waves on the background fluid generates large-scale dynamical structures which can have a radial extent much larger than the overshoot layer. Moreover, although the total flux of angular momentum transported is small, it is nonetheless important on the secular timescales considered for the slow tachocline mod-

els. Thus in this case again, significant modifications to the existing slow tachocline models could be required.

\subsubsection{Boundary conditions}

One of the most difficult problems faced by all tachocline models (including the fast tachocline, see Section 9.3) is the choice of boundary conditions used to describe the convective-radiative interface. The problem is exacerbated in the case of the slow tachocline, where meridional flows play an important role in redistributing angular momentum, preserving the thermal-wind balance and confining the internal field. Indeed, artificial flows generated on the 
boundary of the computational domain are an inevitable consequence of any attempt to impose stresses locally. Two situations may arise.

If the boundary is assumed to be impermeable, Ekman and EkmanHartmann layers form (the layer structure is modified for stress-free boundaries, but does not disappear); numerical models must monitor the amplitude of these boundary layer flows and ensure that they are only a small perturbation to the baroclinic flows of interest. This constraint places upper limits on the values of the Prandtl $(\nu / \kappa)$ and inverse Roberts $(\eta / \kappa)$ numbers. However, even in a limit where Ekman flows can be neglected, the presence of an impermeable outer boundary constrains the geometrical structure of the meridional flow cells by limiting their upper radial extent, and by mass conservation, their latitudinal geometry. This numerical artefact is inevitable in the case of impermeable boundaries, and will affect the latitudinal force balance within the tachocline.

Another option is to relax the condition of impermeability. In that case, continuity of radial stresses replaces the condition of impermeability, but the problem is then merely transposed into a Reynolds stress modelling problem for turbulent convection. In addition, associated with the thought that it is possible to approximate the radiative-convective interface with simple "boundary conditions" is the underlying assumption that the structure and dynamics of the convective region are independent of the tachocline dynamics. However, the recent work of Miesch (2003) and Rempel (2005) refute this hypothesis. The differential rotation near the convective-radiative interface is related to the differential rotation in the convective region, which results from the angular momentum balance between Reynolds stresses and largescale meridional flows; these flows burrow into the tachocline and advect entropy to create a latitudinal entropy gradient which strongly constrains differential rotation through the thermal-wind balance. Thus the radiativeconvective system is inseparably coupled. It is to be hoped that in the next few years, models will pay particular attention to modelling the convective zone and the tachocline simultaneously.

The role of the convection zone as a boundary condition on the magnetic field is even more ambiguous. Even while leaving aside the possible presence of a dynamo field in the outer layers of the tachocline (see Section 9.3 for a review of the effect of the dynamo field on the tachocline dynamics), currently used boundary conditions could be warping our conclusions on the slow tachocline dynamics. All models thus far assume the convective zone to be nearly perfectly insulating $(\eta \rightarrow \infty)$ and match a potential field to the internal field. By assumption, field lines are smoothly anchored to the convective zone (i.e. to the outer boundary). However, we know that 
this is very far from the true situation: overshooting plumes interact with the magnetic field lines, stirring and shaking them, advecting them into large horizontal excursions, promoting reconnection as well as regeneration (the dynamo effect). In fact, it is more likely that the combined effect of convection is to confine the interior field (at least, its long-term averaged component) somewhat below the overshoot region. Indeed, flux expulsion and magnetic pumping by the convective plumes (Tobias et al. 2001; Dorch \& Nordlund 2001) is sometimes thought of as being the principal reason for the lack of overlap between the internal primordial field and the dynamo field (as discussed by Boruta 1996). By contrast, the underlying assumption that field lines can be smoothly anchored into the convective zone leads to the ubiquitous emergence of a Ferraro state of isorotation in most numerical simulations of the slow tachocline. It will be interesting to know whether this conclusion holds should a more realistic model of the effect of the overshooting plumes on the primordial field be used.

In any case, the presence of a dynamo field may entirely change our view of the solar tachocline; the next section reviews recent models which explicitly involve the solar dynamo in the tachocline dynamics.

\subsection{Dynamo field confinement: the fast tachocline}

The solar dynamo field is observed through the regular emergence of strong flux concentrations at the solar surface, which appear in the form of active regions composed of dark sunspots and bright faculae. Chapter 14 reviews current observational knowledge of the solar dynamo and the potential role of the tachocline in its generation. Some important models favour the radiative-convective interface as the optimal location for the solar dynamo (Parker 1993): field stretching by the strong shear in the azimuthal flow can generate large-scale toroidal fields, accumulating in the tachocline until buoyancy instabilities trigger their rise into the convective region. From there, part of the flux emerges coherently through the surface, while the rest is promptly distorted into small-scale fields in all directions. Non-zero mean flow helicity results in non-zero mean poloidal flux generation, which is then pumped back down into the tachocline by convective overshooting. Many alternative models of the solar dynamo exist (Ossendrijver 2003), some in which dynamo action is independent of the tachocline shear and relies only on turbulent and large-scale motions within the convective zone (Glatzmaier 1984; Brun, Miesch \& Toomre 2004; Brandenburg 2005). In these models too, however, magnetic flux is necessarily pumped into the tachocline by overshooting convective plumes (Tobias et al. 2001). 
The inevitable presence of strong dynamo-generated magnetic fields in the tachocline naturally raises many questions: what are the consequences for the tachocline dynamics? how far down into the tachocline does the dynamo field penetrate? could the dynamo field be entirely, or part-responsible for the observed rotation profile below the convective zone?

Contrary to the primordial field confinement models described above, the dynamics arising from the interaction of the tachocline shear with the dynamo field occur on much shorter timescales. The intrinsic field variability is of the order of 11 years, with a much larger amplitude than the assumed primordial field (and a correspondingly much shorter Alfvén time). Shear and magneto-shear instabilities operate on timescales typical of the rotation rate and Alfvén timescales (Chapter 10). Finally, where overshoot is implied, the flow turnover timescale is of the order of a month. For obvious reasons, this new view of the tachocline was loosely called the fast tachocline (Gilman 2000).

\subsubsection{Fast tachocline diffusion models}

How deep is the fast tachocline? A quick answer associates the thickness of the fast tachocline with the dynamo field penetration depth. The dynamo field is pumped into the overshoot layer by downward penetrating plumes (Tobias et al. 2001) and diffuses downward into the radiative zone. However, the regular field polarity reversal plays an important role in limiting the field diffusion, since each cycle nearly cancels out the previous one (Mestel \& Weiss 1987); as a result the field is strongly suppressed within a skin-depth $\delta_{\mathrm{SD}} \sim\left(\tau_{\mathrm{D}} / \tau_{\eta}\right)^{1 / 2} r_{\mathrm{cz}}$ (assuming the dynamo is exactly periodic with a period $\tau_{\mathrm{D}}$ and where $\tau_{\eta}=r_{\mathrm{cz}}^{2} / \eta$ is the Ohmic diffusion timescale). For a laminar tachocline with microscopic diffusivity $\eta \sim 0.04 \mathrm{~m}^{2} \mathrm{~s}^{-1}$ the skin depth is less than a few kilometers. This figure can be increased to a few $\mathrm{Mm}$ should one consider eddy diffusion in a turbulent tachocline (with $\eta_{\mathrm{t}} \sim 10^{6} \mathrm{~m}^{2} \mathrm{~s}^{-1}$ ) (Forgács-Dajka \& Petrovay 2001). Whether turbulence in the tachocline does indeed act as an "eddy diffusivity" should be kept in mind throughout the following section, and is discussed in more detail in Section 9.3.2 and in Chapter 13.

A promising way of confining the tachocline was first suggested and later developed by Forgács-Dajka \& Petrovay (2001) (Forgács-Dajka \& Petrovay 2002; Forgács-Dajka 2004). They consider the structure of a turbulent tachocline pervaded by an oscillatory dynamo field. The field diffuses downward into the radiative zone and interacts with the tachocline shear. By construction, within the dynamo skin-depth the magnetic diffusion timescale is 
of the order of the dynamo period. The Alfvén crossing time, on the other hand, depends on the imposed field amplitude and can be assumed to be much smaller than the dynamo period for fields of the order of $1 \mathrm{~T}$ or larger. Ferraro isorotation along the poloidal field lines is therefore rapidly achieved.

In their first paper on the dynamics of the fast tachocline, Forgács-Dajka \& Petrovay assume a given poloidal field structure within the dynamo skindepth and impose a sheared angular velocity profile at the interface with the convective zone (see equation 9.4). These are equivalent to the assumption that all meridional motions are negligible within the tachocline; indeed, in that case the equations governing the poloidal and toroidal components of the field decouple. The poloidal component satisfies a simple diffusion equation with periodic forcing, which has a spatially damped oscillatory solution. Here for simplicity the poloidal field $B_{p}$ is assumed to have the functional form

$$
B_{p}(r, \theta, t)=\bar{B}_{p}(r, \theta) \cos \left(\omega_{\mathrm{D}} t\right)
$$

where $2 \pi / \omega_{\mathrm{D}}=\tau_{\mathrm{D}}=22 \mathrm{yr}$. Under those conditions, the azimuthal component of the momentum and induction equations can be integrated to obtain the angular velocity profile and toroidal field profile as a function of time.

An approximate analytical solution to the governing equations can be derived in the limit of large poloidal field strength (i.e. in the limit where there is a clear separation between the Alfvén time and the dynamo period), and thin tachocline. Let $v_{\mathrm{A}}$ be the typical Alfvén velocity of the imposed poloidal field; then by assumption $\epsilon=r \omega_{\mathrm{D}} / v_{A} \ll 1$. Following ForgácsDajka \& Petrovay (2001), the equations are for simplicity written in a local Cartesian system (with $\theta \leftrightarrow x$ and $r \leftrightarrow z$ ). In units of the Alfvén timescale and the radius of the convective zone the non-dimensional governing equations are

$$
\begin{aligned}
& \partial_{t} u_{\phi}=\cos (2 \pi \epsilon t) \partial_{x} B_{\phi}+\frac{\tau_{A}}{\tau_{\nu}} \nabla^{2} u_{\phi}, \\
& \partial_{t} B_{\phi}=\cos (2 \pi \epsilon t) \partial_{x} u_{\phi}+\frac{\tau_{A}}{\tau_{\eta}} \nabla^{2} B_{\phi} .
\end{aligned}
$$

In the limit $\epsilon \ll 1$ it is possible to perform a two-timescale analysis and seek solutions on the slow timescale $\tau=\epsilon t$ (which evolves on the timescale of the cyclic dynamo field). The slow solutions satisfy the reduced equation

$$
\begin{aligned}
& \cos (2 \pi \tau) \partial_{x} B_{\phi}=-\frac{\tau_{A}}{\tau_{\nu}} \nabla^{2} u_{\phi}, \\
& \cos (2 \pi \tau) \partial_{x} u_{\phi}=-\frac{\tau_{A}}{\tau_{\nu}} \nabla^{2} B_{\phi},
\end{aligned}
$$

and, should one assume that $\partial_{z} \gg \partial_{x}$, can be found analytically; they display 
an oscillatory temporal structure with the timescale of the imposed field $\tau_{\mathrm{D}}$, and an oscillatory spatially damped structure below the convective-radiative interface on a typical lengthscale $\delta_{D}$ where

$$
\frac{\delta_{\mathrm{D}}}{r}=\left(\frac{4 \tau_{\mathrm{A}}^{2}}{\tau_{\nu} \tau_{\eta} \cos ^{2}(2 \pi \tau) L^{2}}\right)^{1 / 4},
$$

and $L$ is the latitudinal wavenumber of the imposed poloidal field. Not surprisingly, this estimate is equivalent to the depth of a Hartmann layer for an imposed field with field lines parallel to the boundary and amplitude $B_{0} \cos \left(2 \pi t / \tau_{\mathrm{D}}\right)$. This fast tachocline model therefore predicts the same tachocline thickness scalings as a function of the imposed field as had been obtained by Rüdiger \& Kitchatinov (1997) $\dagger$. By extension, there is a natural generalization of the result should the imposed dynamo field have a strong radial component.

For the model assumptions to be consistent, it is important to verify that $\delta_{\mathrm{SD}} \gg \delta_{\mathrm{D}}$. This places lower limits on the imposed field strength for a given turbulent diffusivity. In addition, if the field is much weaker than about $0.1 \mathrm{~T}$, the simple two-timescale analysis fails and interactions between the dynamo forcing and the Alfvén waves could lead to the excitation of modes with new frequencies. This has not been investigated yet.

Numerical solutions have been computed by Forgács-Dajka \& Petrovay (2001) for a dipolar poloidal field of varying amplitude. They show a clear confinement of the imposed latitudinal shear for large enough field strength (typically, $\left|B_{\mathrm{p}}\right| \sim 0.2 \mathrm{~T}$ for $\eta_{\mathrm{t}} \sim 10^{6} \mathrm{~m}^{2} \mathrm{~s}^{-1}$ ). The latitudinal variation of the field amplitude leads to a significant latitudinal variation of the tachocline depth, which is consistent with the above estimates. Observations however reveal only a weak latitudinal variation of the tachocline position and width (Charbonneau et al. 1999) which could in principle set strong constraints on the poloidal field geometry diffusing from the overlying dynamo. As expected also from the analytical analysis, there is a significant temporal variability of the depth and aspect of the tachocline on an 11-yr period (the differential rotation is independent of the field polarity). Both results confirm and quantify common expectations that there must exist some variability in the tachocline angular velocity profile on the dynamo timescale. However, precise helioseismic observations by MDI/SOI on board SoHO have only been available for slightly less than one solar cycle, and so far, little to no tachocline variability on the dynamo timescale has been detected

† Forgács-Dajka \& Petrovay (2001) derive other scaling laws between the confining field strength and the tachocline thickness in the limit where the dynamo frequency is higher (which could be applicable for other stars than the Sun). 
(Corbard et al. 2001). Definite answers on this topic are impatiently expected. Interestingly, 1.3yr torsional oscillations are detected instead (Howe et al. 2000).

In following works, Forgács-Dajka \& Petrovay (2002) and Forgács-Dajka (2004) study various improvements to the model, including the effects of a large-scale (imposed) meridional flow, of a radially varying magnetic diffusivity and varying magnetic Prandtl number. The background state is derived from the solar model of Guenther et al. (1992). They also calculate the poloidal component of the dynamo field self-consistently from the poloidal component of the advection-diffusion equation: in these new simulations, the poloidal field is advected by the imposed meridional flows in addition to diffusion. Finally, they impose a realistic description of latitudinal and temporal variation of the migrating dynamo field as a boundary condition, which is derived from the observations of Stenflo (1994). The modeled meridional flows are poleward near the solar surface with a velocity of about $10-20 \mathrm{~m} \mathrm{~s}^{-1}$, in accordance with direct observations of the motion of small magnetic features (e.g. Komm, Howard \& Harvey 1993) or inferences from local helioseismology (Giles et al. 1997). Two geometries are studied: a single-cell structure with an equatorward return flow in the tachocline, and a double-cell structure with a poleward return flow in the tachocline and a null node at about $r=0.85 r_{\odot}$. Note that numerical simulations of turbulent convection do not appear to favour the view of stable long-lived circulation cells deep in the convective zone; meridional flows are instead very intermittent, with strongly variable geometries (Brun \& Toomre 2002).

The results, illustrated in Figure 9.4, can be summarized as follows. The role of the meridional flows as transporters of angular momentum naturally aids the tachocline confinement process in the case of the modelled twocell circulation pattern (by transporting angular momentum poleward) and hinders it in the case of the single-cell circulation pattern. The numerical simulations confirm these expectations, and suggest that flows as slow as a few $\mathrm{cm} \mathrm{s}^{-1}$ in the tachocline have a significant impact on the observed differential rotation profile.

A strong decrease in turbulent magnetic diffusivity with depth beneath the tachocline is expected from the steep increase in the background stratification. Note that the decrease in turbulent mixing below the tachocline is clearly constrained by independent observations of the light element depletion fraction (see Chapter 2). In that case again, the imposed convection zone shear is still easily quenched by the fast tachocline fields. However, across the turbulent/laminar transition the dynamo field penetration 


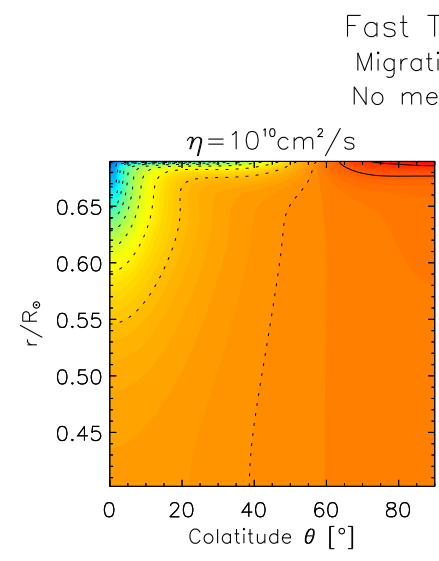

Fast Tachocline Model

Migrating magnetic field

No meridional circulation
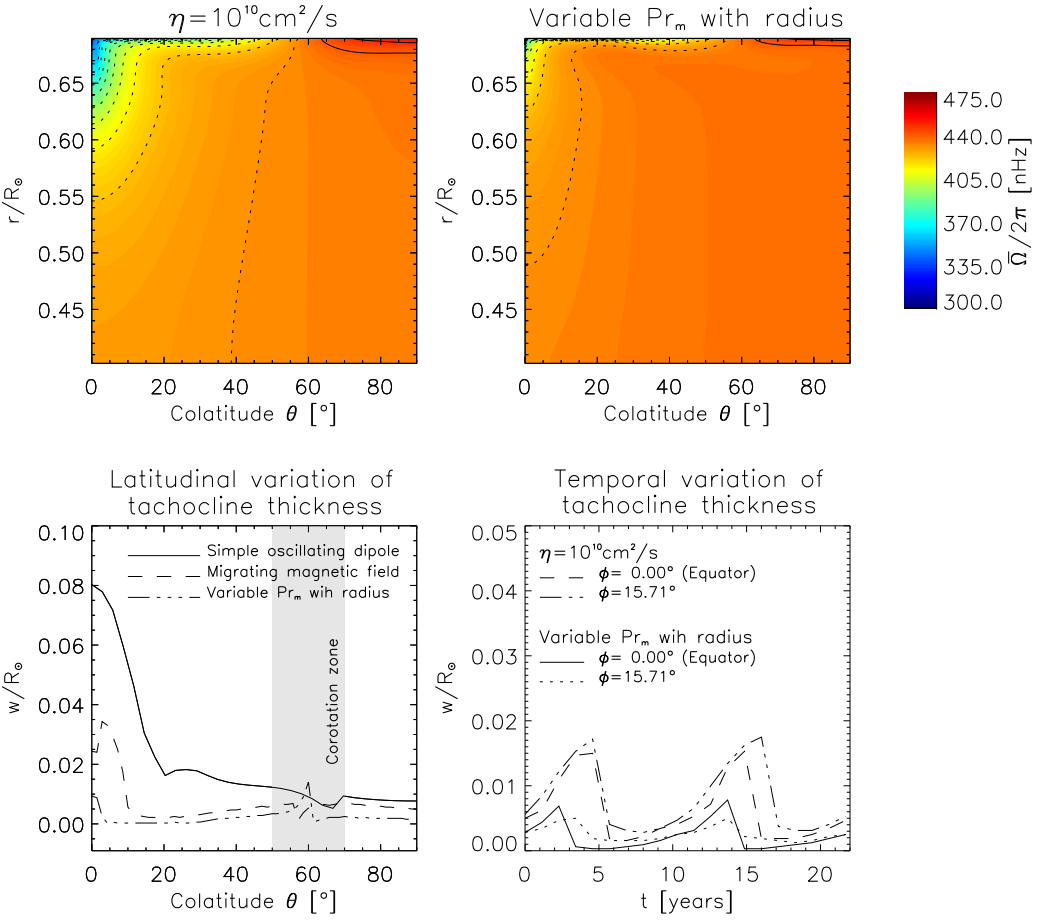

Fig. 9.4. Numerical solutions for the fast tachocline model of Forgács-Dajka \& Petrovay. This simulation includes a realistic representation of the poloidal field extracted from the Butterfly diagram, but neglects meridional motions. Upper panels: Resulting differential rotation spreading into the radiative interior in two cases. In the left-hand panel $\eta=\nu=10^{6} \mathrm{~m}^{2} / \mathrm{s}$ throughout the domain (in which case $\left.P_{m}=\nu / \eta=1\right)$. In the right-hand panel $P_{m}$ is varied with depth between 0.024 and 0.1 . In this case the variations of $\eta$ and $\nu$ are: $\log _{10} \eta=3.5-6$ and $\log _{10} \nu=2-5$. Bottom panels: Corresponding latitudinal variation (left-hand side) and temporal variation (right-hand side) of the tachocline thickness.

is abruptly suppressed and would therefore not be able to reduce any deepseated residual shear related to solar spin-down; this is an intrinsic problem of all fast tachocline models. One possible solution stems from the fact that the solar dynamo cycle is not exactly periodic. Mestel \& Weiss (1987) suggested that the (apparently) random component of the dynamo field could diffuse much deeper into the radiative zone than its periodic counterpart. I investigated this possibility in detail (Garaud, 1999), and found that an 
internal field with an rms value of about $10^{-4} B_{0}$ (where $B_{0}$ is the amplitude of the poloidal component of the dynamo field) could build up deeper in the interior.

\subsubsection{Discussions and prospect for the dynamo confinement model}

In comparison with slow tachocline models, the idea proposed by ForgácsDajka \& Petrovay has the advantage of being based on a robust balance of forces, which holds even in the presence of instabilities (it does in fact rely on the presence of instabilities), and can be tuned to compensate any additional angular momentum transport from convective plumes, gravity waves or meridional flows. The spatial variation of the tachocline depth observed in the numerical simulations can be reconciled with observations for specific poloidal field structures, and the strong temporal variation observed could still be consistent with observations should the tachocline be in fact a little bit shallower than current estimates (this statement is mostly based on the resolution of helioseismic inversions).

One must nonetheless bear in mind the three assumptions inherent in the model: the tachocline is turbulent, the turbulence leads to an eddy diffusivity greater than $10^{5} \mathrm{~m}^{2} \mathrm{~s}^{-1}$ in the tachocline, and finally, the dynamo generation mechanism does not rely on the detailed tachocline structure.

If we assume that the tachocline has a width $\sim 0.02 r_{\odot}$, then turbulent motions at the level required by the fast tachocline model cannot result from overshooting plumes only. The stability of the tachocline to hydrodynamical and magneto-hydrodynamical instabilities was discussed in Section 9.2.5.1 and is reviewed in Chapters 10 and 11; magneto-shear instabilities offer a promising route for the maintenance of turbulent motions. In fact, these instabilities are so ubiquitous that the maintenance of large-scale fields in the tachocline appears to be the more relevant problem. Nonetheless, the first of the three governing assumptions is not much under dispute.

However, the role of turbulent motions in "diffusing" large-scale fields is a far more difficult issue. Although very commonly used in astrophysical MHD models, the physical basis for turbulent diffusivity, as well as its parameterization, are still ambiguous. The concept of turbulent diffusion is derived from heuristic arguments on the vectorial form of the averaged electromotive force due to small-scale fields and flows (see Chapter 14):

$$
\overline{(\mathbf{u} \times \mathbf{b})}_{i}=\alpha_{i j} B_{j}-\beta_{i j k} \partial_{j} B_{k} \ldots
$$

This expression naturally emphasizes the tensorial nature of the turbulent 
diffusivity $\beta$; assuming that $\beta \sim \eta_{\mathrm{t}}$ is a scalar is a largely unjustified (but commonly used) simplification.

The turbulent diffusivity is known to be quenched when the magnetic fields start having a strong effect on the turbulent flow (near energy equipartition); at the largest scales in the tachocline, this effect is relevant for fields upward of a few thousand Gauss, which already has implications for fast tachocline models. But the situation may in fact be much worse. In the tachocline, the magnetic Prandtl number is of the order of $\nu / \eta \sim 10^{-2}$; if a small-scale dynamo indeed operates at these values of the magnetic Prandtl number (Boldyrev \& Cattaneo 2004) magnetic energy accumulates somewhere on the turbulent inertial range and reaches equipartition well before the larger scale field does. This process could quench the turbulent magnetic diffusivity for much lower field strengths (Cattaneo \& Vainshtein 1991). Catastrophic $\eta$-quenching is shown to occur in $2 \mathrm{D}$ flows through numerical simulations (Cattaneo 1994) and quasi-linear closure (Gruzinov \& Diamond 1995). The situation is still unclear in the case of 3D flows. The $\eta$-quenching process could pose serious threats to the fast tachocline models: using the scalings proposed by Cattaneo \& Vainshtein (1991), large-scale fields as low as few Gauss would suffice to quench the turbulent diffusivity of the fast tachocline by several orders of magnitude. This creates an intrinsic contradiction within the model.

In any case, the current fast tachocline model neglects all effects of the turbulent motions except for their role in enhancing the magnetic diffusivity. However, other macroscopic effects are known to occur and are likely to play an important role in the tachocline dynamics. Turbulent flux expulsion has been observed in a wide variety of systems where turbulent and laminar regions coexist (Tao, Proctor \& Weiss 1998; Tobias et al. 2001). Smallscale field generation by turbulent motions, the $\alpha$-effect, has also been predicted by turbulence closure models (Krause \& Rädler 1980) and observed in numerical simulations (Brandenburg et al. 1990). Finally, non-isotropic Reynolds stresses and turbulent Maxwell stresses may be as important as the large-scale Lorentz forces in reducing the imposed shear. In other words, a consistent model for the fast tachocline will require a consistent description of the effects of turbulent motions on the large-scale flows and fields.

Building on this idea, another natural step in the study of fast tachocline confinement models is to calculate self-consistently the temporal evolution of the field and the flow, using for instance a mean-field dynamo model. Indeed, current mean-field models calculate the field evolution assuming a given angular velocity profile in the tachocline, whereas current tachocline confinement models study the effect of an assumed dynamo field on the 
shear. In an integrated model, can dynamo action be sustained if the radial shear is quenched by the dynamo itself? This could indeed happen should dynamo action rely more on the latitudinal shear than the radial shear, or if the solar dynamo is more of an $\alpha^{2}$ dynamo than an $\alpha \Omega$-dynamo. Reproducing simultaneously the tachocline profile and the solar cycle is an interesting challenge which could provide much insight into the correct parameterization of the $\alpha$ - and $\beta$-effects.

\subsection{Discussion and Prospects}

We have now reached a stage in the process of studying the tachocline dynamics where there exist a large enough variety of studies, models and observations to support critical discussions. What are the next steps in the study of the tachocline magnetohydrodynamics? The few points that I believe will have significant impact on our understanding of the tachocline in the next few years are the following.

Coexisting "fast" and "slow" tachoclines? In the light of the discussions outlined in Sections 9.2 and 9.3, is it still possible to consider the idea of coexisting "fast" and "slow" tachoclines? The only way to do this would be to construct a complex layered structure starting from the bottom of the convective zone with a turbulent, magnetic overshoot region, which gradually quietens downward to give way to a more laminar region where the large-scale (dynamo) fields are pumped, stored and stretched. Slightly further down, the very low magnetic diffusivity forbids the oscillating field from penetrating very far down and thus appear successively the well-ventilated, magnetic free region of the Gough \& McIntyre tachocline, the magnetic diffusion layer and finally the magnetically constrained interior. And most of the above must be packed, according to observations, within a total width spanning no more than $2-4 \%$ of the solar radius. This scenario can only work if fluid motions in the tachocline are to a very large degree two-dimensional. However, there are doubts that this may be the case at all times despite the strong stratification. Magnetic buoyancy instabilities (Tobias \& Hughes 2004) are intrinsically three-dimensional, and numerical simulations suggest there are occasional very strong overshooting events with large radial extent. Can the slow tachocline balance survive these mixing events? More precisely if, as suspected, the Gough \& McIntyre model indeed harbours multiple equilibria, mixing events extending between the interior and the overshoot region could dredge out interior field lines and drag them into the convective region, triggering the transition from a confined interior field to 
the open field configuration. Should this happen, there is no simple mechanism capable of reverting the system to the confined field configuration of the Gough \& McIntyre model within the typical timescale of occurence of the mixing events.

The role of the interaction between overshooting plumes and an internal primordial field. As discussed in Section 9.5.2.3, this interaction is likely to play a dominant role in the tachocline dynamics. Tamara Rogers and I have begun studying this phenomenon to determine whether this may indeed be a sufficient, self-consistent way of confining an internal field while bypassing the need for baroclinic meridional flows. We hope to show for instance that the ubiquitous emergence of Ferraro rotation in laminar models is in fact an artefact of the simplified interface conditions; in fact, we believe that the interaction between overshoot and an internal field may form the basis for a minimalist model of the tachocline and the radiative interior.

The role of gravity waves. Talon \& Charbonnel (2005) have recently claimed that the continuous adjustment of the angular velocity of the radiative core to that of the convection zone could in fact be entirely attributed to gravity wave mixing. This would supress the need for an internal primordial field. An important task for the near future is to test the Talon \& Charbonnel model for angular momentum transport against direct numerical simulations of gravity waves in the solar interior (Rogers \& Glatzmaier 2006), and to investigate ways in which observations (combining asteroseismology, surface light-element abundances and magnetic activity measurements) may help distinguish between the magnetic and non-magnetic scenarios.

Consequences of recent solar abundance revisions for all tachocline models. The recent announcements of revised solar abundances of carbon, nitrogen and oxygen (Allende Prieto et al. 2001; Asplund et al. 2005) could entirely re-shape our view of the solar interior. Revised solar models with similar total mass, luminosity and age predict a significantly shallower convection zone than previous estimates (Bahcall et al. 2004), which are in serious disagreement with helioseismic inversions (Christensen-Dalsgaard et al. 1991). What are the consequences of these findings for tachocline models? If indeed the solution is related to opacity calculations - see Chapter 2 (which is quite plausible, Bahcall et al. 2005; Drake \& Testa 2005) then the tachocline models will, on the whole, be unchanged. However, if the position of the critical radius for convective stability is indeed raised to $r=0.726 r_{\odot}$, then most existing tachocline models are affected. To begin 
with, the standard solar model will have to be revised to explain the serious sound speed discrepancy between models and observations. Perhaps the addition of an extended nearly-adiabatic region matching smoothly on to the strongly stratified interior near $r=0.713 r_{\odot}$ will suffice, but can overshoot models explain the subsistance of such a large region throughout the solar lifetime as well as the heat fluxes required to operate a smooth matching $\dagger$ ? If so, will this be more likely to accomodate both fast and slow tachoclines? Is there indeed a relation between the tentatively observed variation with latitude of the tachocline depth (with a thicker tachocline near the poles, Basu \& Antia 2003) and that of the overshoot region (likewise, Brummell, Clune \& Toomre 2002)?

The early evolution of the Sun and its relation to the internal primordial field. Given its likely dominant role in the interior dynamics, it is perhaps surprising that we know so little about the interior field. How much of the collapsing cloud magnetic flux survives the fully convective phase of stellar evolution? What happens to this flux as the convective zone finally retreats (see Chapter 8)? The Mount Wilson observations of the magnetic activity of very young solar type stars now permit a more comprehensive study of the correlation between dynamo action, rotation and internal structure: can we construct a model of the early solar magnetism that would include this new data and enable us to predict the current internal field strength and geometry?

Self-consistent mean-field hydrodynamics and dynamo models. Current mean-field dynamo models assume a given differential rotation profile, while current fast tachocline models assume a given magnetic field profile. Rempel (2005) showed that it is now possible to use mean-field hydrodynamics to model simultaneously the tachocline and the convection zone; the extension of this work to include magnetic stresses as well as mean-field dynamo action would provide the first self-consistent model of rotation and dynamo action in the Sun. This would be a tremendous advance in the field, since the self-consistent determination of rotation (which can be measured by helioseismology) and meridional flows (which appear to constrain the equatorward sunspot drift throughout the cycle in many types of dynamos) may help distinguish between various competing dynamo models. Comparison with the rotation profile and magnetic activities of other stars would also help refine our understanding of this exceedingly complex system. In fact,

$\dagger$ A large discontinuity in $N^{2}$ at the base of the convection zone is precluded by helioseismology (Basu 1997). 
such an approach may be the only route towards a better understanding of interior dynamics: it is becoming increasingly clear that we have very little hope of reaching the asymptotic values of the Reynolds and Rayleigh numbers in 3D simulations of the whole Sun that would permit a trustworthy study of the convection zone and the tachocline. However, numerical simulations in a local box are on the other hand much closer to solar values, and may help constrain the parameterizations to be used in mean-field models.

\section{Acknoledgements}

I thank all of the Isaac Newton Meeting participants for enlightening and stimulating discussions about this fabulous subject. The completion of this

manuscript would not have been possible without the help and support of Nic Brummell and Douglas Gough. I also thank Fausto Cattaneo, Gary Glatzmaier, Chris Jones, Michael McIntyre, Bob Rosner, Steve Tobias and Nigel Weiss for clarifying many of the complex scientific issues discussed here.

\section{References}

Acheson, D. J. \& Hide, R. (1973). Rep. Prog. Phys., 36, 159.

Balbus, S.A. \& Hawley, J.F. (1994). Astrophys. J. 266, 769.

Basu, S. (1997). Mon. Not. Roy. Astron. Soc. 588, 572.

Boldyrev, S. \& Cattaneo, F. (2004). Phys. Rev. Lett. 92

Boruta, N. (1996). Astrophys. J. 458, 832.

Boyer, D.W. \& Levy, E.H. (1984). Astrophys. J. 277, 848.

Braithwaite, J. \& Spruit, H.C. (2004). Nature 431, 819.

Brandenburg, A. (2005). Astrophys. J. 625, 539.

Brummell, N.H., Clune, T.L., \& Toomre, J. (2002). Astrophys. J. 570, 825.

Brun, A.S. \& Toomre, J. (2002). Astrophys. J. 570, 865.

Brun, A.S., Miesch, M.S. \& Toomre, J. (2004). Astrophys. J. 614, 1073.

Brun, A.S. \& Zahn, J.-P. (2006). Astron. Astrophys. submitted

Cally, P.S., Dikpati M. \& Gilman (2003). Astrophys. J. 582, 1190.

Cattaneo, F. \& Vainshtein S.I. (1991). Astrophys. J. 376, L21.

Cattaneo, F. (1994). Astrophys. J. 434, 200.

Charbonneau, P., Dikpati, M. \& Gilman, P.A. (1999). Astrophys. J. 528, 523.

Charbonneau, P. et al. (1999). Astrophys. J. 527, 445.

Christensen-Dalsgaard, J., Gough D.O. \& Thompson, M.J. (1991). Astrophys. J. $\mathbf{3 7 8}, 413$.

Clune T.L. et al. (1999). Parallel Comp. 25, 361.

Corbard, T. et al. (2001). in Proceedings of the SOHO 10/GONG 2000 Workshop: Helio- and astero-seismology at the dawn of the millennium. ed A. Wilson (ESA Publications), p. 265.

Cowling, T.G. (1945). Mon. Not. Roy. Astron. Soc. 105, 166.

Cowling, T.G. (1957). in Magnetohydrodynamics, Interscience, New York. 
Dikpati, M. \& Gilman, P.A. (2001). Astrophys. J. 551, 536.

Dormy, E., Cardin, P., \& Jault, D. (2001). Earth Planet. Sci. Let. 160, 15.

Dormy, E., Jault, D. \& Soward, A.M. (2002). J. Fluid Mech. 462, 263.

Elliott, J.R. \& Gough, D.O. (1999). Astrophys. J. 516, 475.

Ferraro, V.C.A. (1937). Mon. Not. Roy. Astron. Soc. 97, 458.

Forgács-Dajka, E. \& Petrovay, K. (2001a). Sol. Phys. 203, 195.

Forgács-Dajka, E. \& Petrovay, K. (2002). Astron. Astrophys. 389, 629.

Forgács-Dajka, E. (2004). Astron. Astrophys. 413, 1143.

Garaud, P. (1999). Mon. Not. Roy. Astron. Soc. 304, 583.

Garaud, P. (2001). Mon. Not. Roy. Astron. Soc. 324, 68.

Garaud, P. (2002). Mon. Not. Roy. Astron. Soc. 335, 707.

Giles, P.M. et al. (1997). Nature 390, 52.

Gilman, P.A. \& Fox, P.A. (1997). Astrophys. J. 484, 439.

Gilman, P.A. \& Fox, P.A. (1999a). Astrophys. J. 510, 1018.

Gilman, P.A. \& Fox, P.A. (1999b). Astrophys. J. 522, 1167.

Gilman, P.A. \& Dikpati, M. (1999). Astrophys. J. 512, 417.

Gilman, P.A. \& Dikpati, M. (2000). Astrophys. J. 528, 552.

Gilman, P.A. (2000). Sol. Phys. 192, 27.

Glatzmaier, G.A. (1984). J. Comp. Phys. 55, 461.

Glatzmaier, G.A. (1985). Astrophys. J. 291, 300.

Gough, D.O., \& McIntyre, M.E. (1998). Nature 394, 755.

Gruzinov, A.V. \& Diamond P.H. (1995). Phys. Plasmas 2, 1941.

Günther, D.B. et al. (1992). Astrophys. J. 387, 372.

Howe, R. et al. (2000). Science 287, 2456.

Kim, E.J., \& MacGregor, K.B. (2001). Astrophys. J. 556, L117.

Kim, E.J., \& MacGregor, K.B. (2003). Astrophys. J. 588, 645.

Komm, R.W., Howard, R.F. \& Harvey, J.W. (1993). Sol. Phys. 147, 207.

Kumar, P. \& Quataert, E.J. (1997). Astrophys. J. 479 L51.

Kumar, P., Talon, S. \& Zahn, J.-P. (1999). Astrophys. J. 520, 859.

MacGregor, K.B., \& Charbonneau, P. (1999). Astrophys. J. 519, 911.

Markey, P. \& Tayler, R.J. (1973). Mon. Not. Roy. Astron. Soc. 163, 177.

Markey, P. \& Tayler, R.J. (1974). Mon. Not. Roy. Astron. Soc. 168, 505.

McIntyre, M.E. (2003). in Stellar astrophysical fluid dynamics, ed. M.J. Thompson \& J. Christensen-Dalsgaard (Cambridge University Press, Cambridge), p. 111.

Mestel, L. (1953). Mon. Not. Roy. Astron. Soc. 113, 716.

Mestel, L. \& Weiss, N.O. (1987). Mon. Not. Roy. Astron. Soc. 226, 123.

Mestel, L., Tayler, R.J. \& Moss, D.L. (1988). Mon. Not. Roy. Astron. Soc. 231, 873.

Miesch, M.S. et al. (2000). Astrophys. J. 532, 596.

Miesch, M.S. (2003). Astrophys. J. 586, 663.

Miesch, M.S. \& Gilman P.A. (2004). Astrophys. J. 611, 268.

Miesch, M.S. (2005). Living Reviews in Solar Physics, 2, 1.

Ossendrivjer, M.A.J.H. (2003). Astron. Astrophys. Rev. 11, 287.

Parker, E.N. (1981). Geophys. Astrophys. Fluid Dyn. 18, 175.

Parker, E.N. (1993). Astrophys. J. 407, 342.

Petrovay, K. (2003). Sol. Phys. 215, 17.

Pitts, E. \& Tayler, R.J. (1985). Mon. Not. Roy. Astron. Soc. 216, 139.

Rempel, M. (2005). Astrophys. J. 622, 1320.

Ringot, O. (1998) Astron. Astrophys. 335, L89.

Rogers, T.M. \& Glatzmaier, G.A. (2005). Astrophys. J. 620, 432. 
Rogers, T.M. \& Glatzmaier, G.A. (2006a). Astrophys. J. submitted

Rogers, T.M. \& Glatzmaier, G.A. (2006b). Astrophys. J. in prep 317, 790.

Rüdiger, G. \& Kitchtinov, L.L. (1997). Astron. Nachr. 318, 273. 375, 149.

Saar, S.H. et al. (1994). in The Eighth Cambridge Workshop on Cool Stars, Stellar Systems and the Sun. ed J.-P. Caillault. Astron. Soc. Pac. Conf. Ser. 64, p.468.

Schatzman, E., Zahn, J.-P. \& Morel, P. (2000). Astron. Astrophys. 364, 876.

Schüssler, M. (1975). Astron. Astrophys. 38, 263.

Schüssler, M., Caligari, P., Ferriz-Mas, A. \& Moreno-Insertis, F. (1994). Astrophys. J. 422, 652 .

Skumanich, A. (1972). Astrophys. J. 171, 565.

Spiegel, E.A. \& Zahn, J.-P. (1992). Astron. Astrophys. 265, 106.

Stenflo, J.O. (1994). in Solar Surface Magnetism ed R.J. Rutten \& C.J. Schrijver, Kluwer Academic Publishers.

Talon, S., Kumar, P. \& Zahn, J.-P. (2002). Astrophys. J. 594, L175.

Talon, S. \& Charbonnel, C. (2005).

Tao, L., Proctor, M.R.E \& Weiss, N.O. (1998). Mon. Not. Roy. Astron. Soc. 300, 907.

Tobias, S.M. et al. (2001). Astrophys. J. 549, 1183.

Tobias, S.M. \& Hughes, D.W. (2004). Astrophys. J. 603, 785.

Tobias, S.M. (2005). in Fluid Dynamics and Dynamos in Astrophysics and Geophysics, ed A.M. Soward, C.A. Jones, D.W. Hughes \& N.O.Weiss (CRC Press, London), p.193.

Watson, M. (1981). Geophys. Astrophys. Fluid Dyn. 161, 285.

Wright, G.A.E. (1973). Mon. Not. Roy. Astron. Soc. 162, 339.

Zahn, J.-P., Talon, S. \& Matias, J. (1997). Astron. Astrophys. 322, 320. 